\title{
TWins in SPACE: ReVIEW AND PeRspectives
}

\section{Carole Tafforin ${ }^{1}$, Nancy L. Segal ${ }^{2}$}

${ }^{1}$ Ethospace, Research and Study Group in Human and Space Ethology, Toulouse, France 2Psychology Department and Twin Studies Center, California State University, Fullerton, USA

ethospace@orange.fr

\begin{abstract}
Monozygotic (MZ) twins subjected to different environmental exposures offer an informative, naturally occurring, co-twin control design. Given their genetic identity, differences in physical, medical and behavioral outcomes between them can be associated with their experiential differences. In space research, a wide range of ethological studies has been conducted on the effects of microgravity on sensorimotor activity and on social group behavior during orbital flights, in a large array of isolated and confined environments and for short-term, mid-term and long-term missions. The study of MZ twin astronaut, Scott Kelly (SK), who spent nearly one-year at the International Space Station (ISS) while his co-twin, Mark Kelly, stayed on earth, is a unique opportunity to identify factors affecting astronauts' health and the extent to which a space mission modifies different adaptive systems at the genetic and epigenetic levels.
\end{abstract}

Keywords: International Space Station, monozygotic, genetic effects, epigenetic effects, adaptation.

${ }^{*}$ Paper presented at XXIV Biennial Conference of Human Ethology 2018, in Santiago, Chile. 


\section{INTRODUCTION}

In twin research, monozygotic (MZ or identical) co-twins subjected to different environmental exposures offer an informative, naturally occurring, co-twin control design. Given their genetic identity, co-twin differences in physical, medical and behavioral outcomes can be associated with their experiential differences. This scientific advantage helps identify factors that either exacerbate or mitigate cognitive performance, mental health, disease risk and other human abilities and conditions (Segal, 2012). This information is critical for its potential applicability to managing social isolation and occupational stress.

Studies of MZ and DZ (dizygotic or fraternal) twins reared apart show that individual differences in special skills are influenced by genetic factors, ranging from .67 (perceptual speed) to .79 (verbal abilities), with the exception of content memory for which genetic influence is estimated to be .33 (Johnson et al., 2007). Twin studies also show genetic effects (.48) on loneliness (Boomsma, Willemsen, Dolan, Hawkley \& Cacioppo, 2005).

Prior studies of non-twins suggest that radiation and microgravity associated with space travel negatively affect some cognitive functions in humans and non-humans (COSPAR, 2004; Grabherr \& Mast, 2010) and increase stress-related disease risk (Pierson, Stowe, Phillips, Lugg \& Mehta, 2005). However, a twin-based approach to these space-related questions that includes a perfect genetic control has never been undertaken.

\section{Twins Reared Apart and Together}

As indicated above, reared-apart twins are a powerful research design, given that the same genotypes are exposed to different environments, revealing possible differences in gene expression. In contrast, reared-together twins experience the same environments, but the experimental exposure of the two co-twins to different environments can be conducted in a relatively controlled manner, as in the case of the Kelly twins, shown in Figure 1. SK spent an extended period of time in the International Space Station, while his co-twin remained on earth. It is unlikely that such a rare opportunity involving MZ
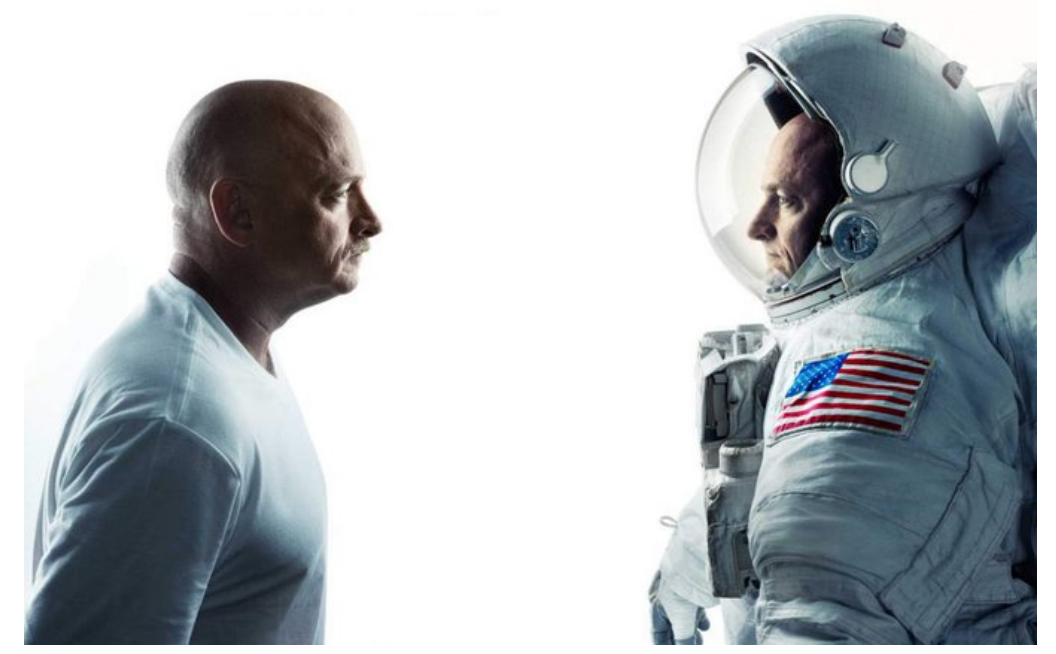

Figure 1: Mark Kelly (left) and Scott Kelly (right). (Picture from @ StationCDRKelly) 
twins will arise again. Interestingly, however, in April 1972, MZ twin astronaut Charles Duke participated in Apollo 16, becoming the tenth individual (and only twin) to reach the moon's surface. His twin brother, William, was born with a heart defect, so was physically unable to pursue a career in space travel; instead, he became a physician (Segal, 2000). A formal comparison of these twins was never undertaken, but would have been far less informative than that of the Kelly twins, due to their different health histories and because Apollo 16 lasted for only eleven days.

\section{Space Travel: Effects On Adaptive Systems}

In space research, a wide range of ethological studies has been conducted on the effects of microgravity on humans during orbital flights (Space shuttle and Mir station) and over time. The first investigations focused on changes in spatial and motor skills within short sequences of reduced gravity from parabolic flights. Experimental protocols were designed for comparing novices, experienced subjects and astronauts. Further analyses showed significant modifications in movement, posture and orientation throughout mid-term mission, from ground training to post-flight periods (Tafforin, 1994).

With respect to these behavioral domains, investigating MZ twin astronauts, SK, who spent nearly one-year aboard the International Space Station (Fig. 2) and his twin brother MK, who remained on earth as a control subject, is a unique opportunity to collect multidisciplinary data on long-term processes. In Spring 2015, SK launched to the ISS, serving as Flight Engineer for the 43rd and 44th expeditions, and as Commander for the 45th and 46th expeditions. During the 340-day mission, almost 400 experiments were conducted on the station. SK landed on March 1, 2016.

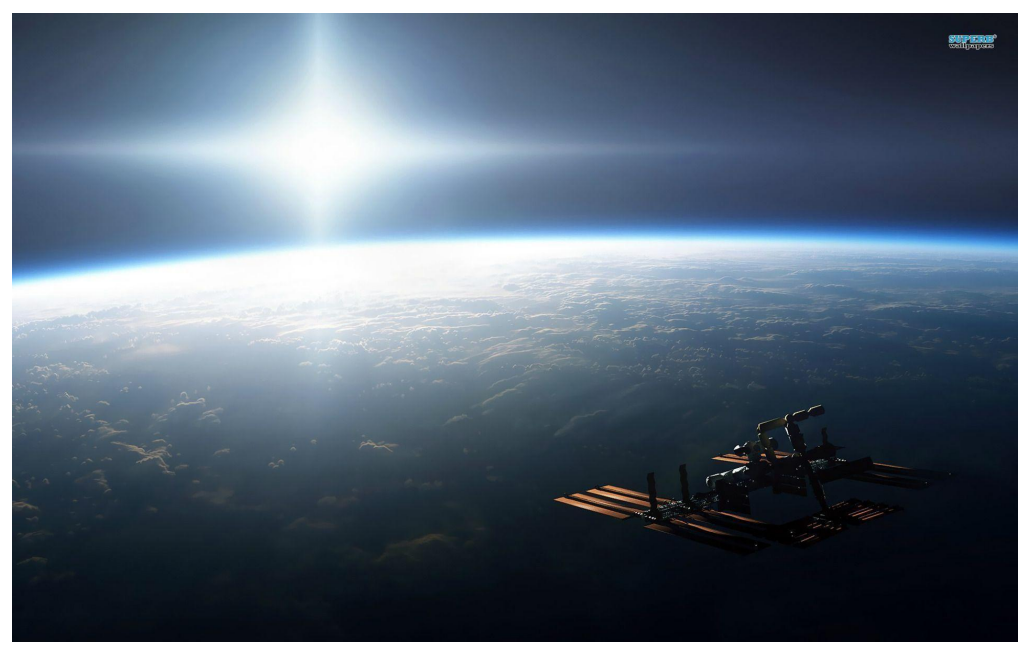

Figure 2: The International Space Station in orbit. (Picture from@rndTYMT-iss-wallpaper) 
The present review documents some of the accumulating discoveries of factors affecting SK's global health, and the extent to which a space mission modifies different adaptive systems-from chemical modifications to cognitive functions. Perspectives regarding functions impacted by epigenetic effects over the long-term and upon changing environments, in space or on earth, are summarized.

\section{REVIEW OF FINDINGS}

Ten groups of investigators (Susan Bailey, Brenda Rana, Suart Lee, Fred Turek, Emmanuel Mignot, Scott M. Smith, Andy Feinberg, Chris Mason, Mathias Basner and Mike Synder) are examining a wide variety of data concerning the Kelly twins' health. The health-related domains of interest include the biomedical profile, cognitive performance, immunological response, bone formation, multi-omics markers, gut microbiome and how DNA might be affected by microgravity and by living in space. Some preliminary findings (IWS, 2017; IWS, 2018), summarized here, show changes as a result of the co-twin control study.

Perhaps the most interesting and provocative finding is that genetic expression is sensitive to changing environments in space and on earth. In particular, SK's telomeres showed elongation while in space, but decreased in length within 48 hours after returning to earth. It is estimated that $7 \%$ of his genes may have changed their expression in space and according to time. However, the question of whether or not real genetic change occurred has been a matter of some debate. Bailey, one of the collaborators on the project, had predicted that SK's telomeres would shorten, given that this is a sign of aging and would reflect the physical stressors of space travel. However, the opposite occurred and the telomeres of other astronauts have shown similar responses. This finding led Bailey to speculate that short telomeres, sensitive to environments in space, might have disappeared, artificially boosting the number of longer telomeres present. Alternatively, she proposed that microgravity may have stimulated release of the enzyme telomerase that could possibly have added length to the telomeres; however, she noted that the lengthening of telomeres has never been demonstrated in humans (Koren, 2017).

Changes were also observed in genes that control functions related to DNA repair, bone formation, gut bacteria digestion and the immune system. It appears that astronauts' global health is also affected as a result of long-term space travel. An integrated publication of the results (Garrett-Bakelman et al., 2019) showed that SK has greater change in his microbiome than his twin brother, but the diversity was basically unaltered. Epigenetic alterations between the twins were also not marked. Cardiovascular changes that are characteristic of other astronauts, were found in SK, but not in MK, such as a $10 \%$ increase in cardiac output, and a modest decrease in blood pressure. SK's carotid artery thickness also increased and stayed that way for four days after his return to earth. SK's cognitive skills were mostly during his post-flight period, in that his performance speed fell for all mental ability tests, excerpt for Digit Symbol Substitution. In addition his accuracy dropped in all areas except for spatial orientation; these declines persisted for a half-year period after his return to earth. 


\section{DISCUSSION}

Subsequent research on the genetic and physiological effects of extended space travel will offer important new perspectives and insights as scientists look toward a long-term journey to Mars. A round-trip journey will minimally require a 6-month outward trip and a 6-month return trip, while a stay on the red planet could last for one year and beyond. Synergies of genetic and epigenetic changes will impact human behavior at both the individual level and at the social level.

The cultural value of the international team-members having to live in micro-society (Tafforin \& Giner Abati, 2017) far from earth may shed light on some aspects of human behavior in an evolutionary context. The assumption is that genetic expression resulting from a weightlessness environment, through adaptive behavioral strategies, will yield a new cognitive representation of the members of a space team. This has been shown in previous research. For the last quarter of a century, a wide range of ethological studies has been conducted on the effects of microgravity on humans during orbital flights (Space shuttle and Mir station) for short-term and mid-term missions. Changes in spatial and motor skills as behavioral strategies, affected by gravity variations from parabolic flights, were key goals of these studies (Tafforin, 1994). In the theoretical model, the adaptive process began with a spontaneous phase showing sensorimotor reflexes, followed by preliminary and integrative phases enhancing specific cognitive functions over the course of space travel with the prevalence of visual cues.

Tying this line of work to twin studies can prove valuable. Identical twin comparisons involving differential exposure to spaceflight factors will further our knowledge of space flight outcomes, as shown above. Secondly, observations were made during a twelve-month polar mission (Concordia station), then during the Mars-500 experiment with reference to the effects of a 520-day period of isolation and

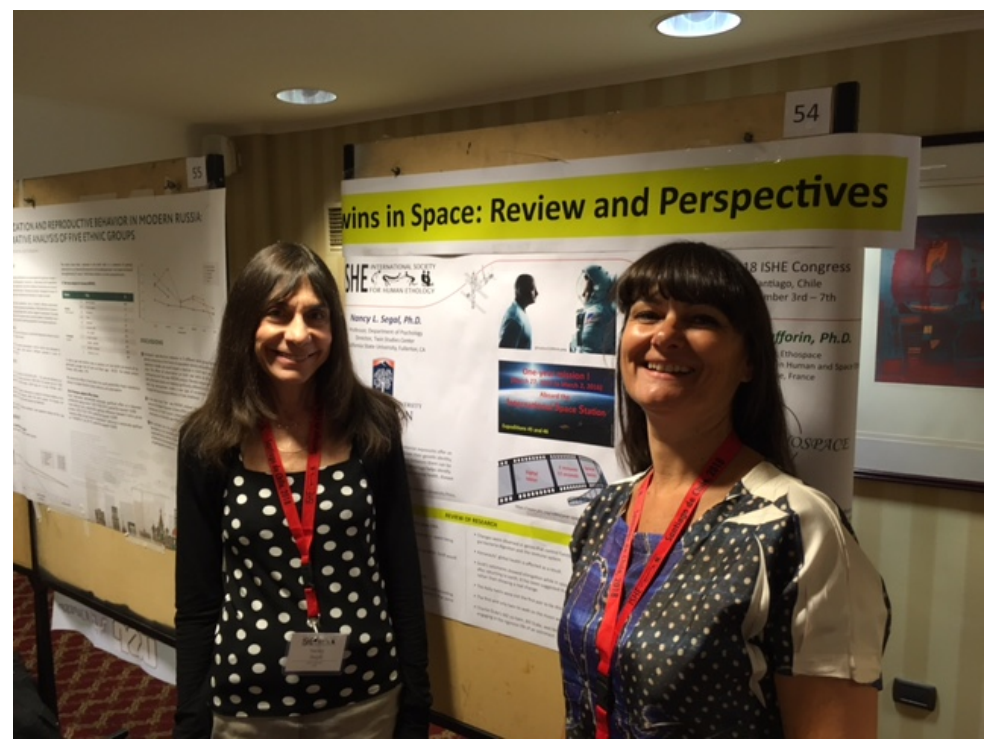

Figure 3: Nancy L. Segal (left) and Carole Tafforin (right) at their poster presentation, ISHE Conference, September 5, 2018, Santiago de Chile. 
confinement (Tafforin, 2013a). In a long-term adaptive process, analyses revealed time effects, cultural preferences and individual differences in crew behavior, simulating a Mars mission (Tafforin, 2013b). Such data constitute a comprehensive database against which to evaluate the results from future studies involving long-term space travel, such as the recent experience of SK. The fact that two close brothers (SK and MK) were separated for nearly a year and SK was in a risky environment raises key questions for these twins, in particular, and for all astronauts and their family members, in general. Particularly, how does prolonged isolation from close family members and socialization with unrelated team members affect psychological and biological systems? Importantly, twin data can further the goal of assessing genetic factors underlying physical, physiological and behavioral aspects of a space exploration mission from an evolutionary perspective.

\section{ACKNOWLEDGMENTS}

The space ethological research of Carole Tafforin as a whole is supported by the French Space Agency. The research on twins of Nancy Segal was supported by senior faculty research awards from California State University, Fullerton.

\section{REFERENCES}

Boomsma, D.I., Willemsen, G., Dolan, C. V., Hawkley, L. C., \& Cacioppo, J. T. (2005). Genetic and environmental contributions to loneliness in adults: The Netherlands Twin Register Study. Behavior Genetics, 35(6), 745-752. DOI

COSPAR (2004). Exposure to radiation accelerates normal brain aging and produced deficits in spatial learning and memory. 35 th COSPAR Scientific Assembly, Paris, France.

Garrett-Bakelman, F. E., Darshi, M., Green, S. J., Gur, R. C., Lin, L., Macias, B. R., ... \& Piening, B. D. (2019). The NASA Twins Study: A multidimensional analysis of a year-long human spaceflight. Science, 364(6436). DOI

Grabherr, L. \& Mast, F.W. (2010). Effects of microgravity on cognition: The case of mental imagery. Journal of Vestibular Research, 20(1,2), 53-60. DOI

IWS (2017). Annual NASA Human Research Program Investigators' Workshop. January 23-26, 2017. Galveston Texas-USA.

IWS (2018). Annual NASA Human Research Program Investigators' Workshop. January 22-25, 2018. Galveston Texas-USA.

Johnson, W., Johnson, W., Bouchard Jr, T. J., McGue, M., Segal, N. L., Tellegen, A., Keyes, M., \& Gottesman, I. I. (2007). Genetic and environmental influences on the VerbalPerceptual-Image Rotation (VPR) model of the structure of mental abilities in the Minnesota Study of Twins Reared Apart. Intelligence, 35(36), 542-562. DOI 
Koren, M. (3. March, 2017). What's Different About Astronaut DNA? The Atlantic, https:// www.theatlantic.com/science/archive/2017/03/nasa-twins-scott-kelly/518145/

Pierson, Stowe, Phillips, Lugg, \& Mehta (2005). Epstein-Barr virus shedding by astronauts during space flight. Brain, Behavior, and Immunity, 19(3), 235-242. DOI

Segal, N.L. (2012). Born together - reared apart: The landmark Minnesota twin study. Cambridge, MA: Harvard University Press. DOI

Segal, N.L. (2000). Entwined lives: Twins and what they tell us about human behavior. New York NY: Plume.

Tafforin, C. (1994). Synthesis of ethological studies on behavioral adaptation of the astronaut to flight conditions. Acta Astronautica, 32(2), 131-142. DOI

Tafforin, C. (1996). Initial moments of adaptation to microgravity of human orientation behavior, in parabolic flight conditions. Acta Astronautica, 38(2), 963-971. DOI

Tafforin, C. (2013a). The MARS-500 crew in daily life activities: An ethological study. Acta Astronautica, 91, 69-76. DOI

Tafforin, C. (2013b). Time effects, cultural preference and individual differences in crew behavior during MARS-500 experiment. Aviation, Space, and Environmental Medicine, 84(10), 1082-1086. DOI

Tafforin, C. \& Giner Abati, F. (2017). Cultural ethology as a new approach of interplanetary crew's behavior. Acta Astronautica, 139, 102-110. DOI 